Im „Journal Club" werden Originalarbeiten aus der internationalen Fachliteratur referiert und kommentiert.

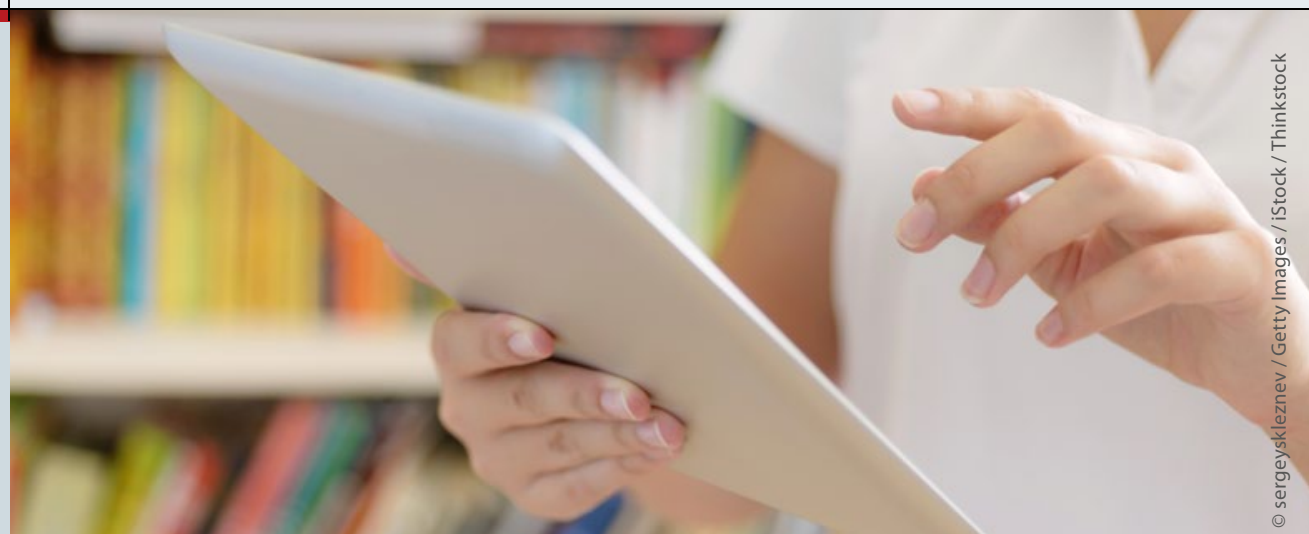

\section{Gefährliche Folgen: Antibiotikaeinsatz im Pflegeheim}

\begin{abstract}
Wie häufig Pflegeheimbewohner Antibiotika erhalten, hängt offenbar stark von der jeweiligen Einrichtung ab; das legt eine Studie aus Kanada nahe. In Heimen mit häufigem Einsatz stieg die Gefahr antibiotikaassoziierter Schäden deutlich - und zwar nicht nur bei den Behandelten.
\end{abstract}

Eiventer ne erstaunliche Variabilität in der Verordnung von Antibiotika an Pflegeheimbewohner zeigt sich in einer kanadischen Studie: Wie die Forscher um Dr. Nick Daneman von der Universität Toronto berichten, schwankte der Antibiotikaeinsatz zwischen den verschiedenen Einrichtungen um das knapp Zehnfache: So lag der Anteil der Antibiotikatage in den Einrichtungen mit niedrigstem Verbrauch bei 20,4, in denen mit höchstem Verbrauch bei 192,9 Tagen, jeweils bezogen auf 1000 Bewohnertage.

An der offenen Longitudinalstudie waren 110.656 Bewohner aus insgesamt 607 Einrichtungen im kanadischen Ontario beteiligt; das Durchschnittsalter lag bei 85 Jahren. Insgesamt wurden an 2.783.000 von 50.953.000 Bewohnertagen Antibiotika verabreicht; dies entspricht einer Rate von 55 Antibiotikatagen pro 1.000 Bewohnertage.

Je großzügiger die Einrichtungen beim Verteilen von Antibiotika waren, desto mehr Schaden richteten diese bei den Patienten an: Die Wahrscheinlichkeit irgendeines unerwünschten Ereignisses im Zusammenhang mit Antibiotika war in den Heimen mit hohem Verbrauch um $24 \%$ höher als bei solchen mit niedrigem Verbrauch. In Ersteren traten antibiotikaassoziierte Durchfälle oder
Gastroenteritiden bei 11,6\% der Bewohner auf, Infektionen mit resistenten Erregern bei $1,3 \%$, Infektionen mit Clostridium difficile bei $0,9 \%$ und Antibiotikaallergien bei $0,1 \%$. Insgesamt lag der Anteil der Schadwirkungen durch Antibiotika bei 13,3\% in Heimen mit hohem Verbrauch gegenüber $11,4 \%$ in solchen mit niedrigem Verbrauch.

Faktoren, die nach Angaben der Forscher die Vulnerabilität für solche Schäden erhöhten, waren u. a. fortgeschrittenes Alter, Diabetes mellitus, eine periphere Gefäßerkrankung sowie Leberund Nierenerkrankungen.

Penicillin war in allen Einrichtungen das am häufigsten verabreichte Antibiotikum, mit einem Anteil an sämtlichen Antibiotikaverschreibungen zwischen $32,6 \%$ und 33,7\%, gefolgt von Fluorchinolonen der zweiten Generation $(27,1 \%$ bis $31,7 \%)$. Einen geringeren Anteil machten Cephalosporine, Nitrofurantoin und Makrolide aus (zwischen 4\% und 9\%).

Frappierend und auch für Pflegebedürftige in Deutschland relevant ist vor allem ein Ergebnis: In den Heimen, die Antibiotika am großzügigsten einsetzten, zeigten nicht nur Antibiotikanutzer deutlich höhere Raten unerwünschter Ereignisse. Die mit der Therapie assoziierten Gesundheitsschäden waren in solchen Einrichtungen auch bei den Be- wohnern signifikant erhöht, die gar keine Antibiotika erhalten hatten $(9,9 \%$ gegenüber $8,7 \%$ in den Heimen mit geringstem Verbrauch).

Die Forscher erklären dies mit einem gesteigerten Selektionsdruck auf das lokale Mikrobiom: Bei hohem Antibiotikaeinsatz steigt in der ganzen Einrichtung die Rate resistenter Keime; vor allem C. difficile kann sich stark vermehren. Da des Öfteren Patienten aus Pflegeheimen in andere Einrichtungen, z.B. Akutkliniken, verlegt werden, besteht nach Daneman et al. außerdem die Gefahr, dass sich resistente Erreger über die Mauern des Heims hinweg ausbreiten.

Die Wissenschaftler um Daneman appellieren an alle Pflegeeinrichtungen, die Prinzipien der Antibiotic Stewardship, also des sorgsamen Umgangs mit Antibiotika, zu befolgen. Die Kernpunkte sind u.a. auf der Seite der CDC (Centers for Disease Control and Prevention) einzusehen. Eine umfassende Anleitung zum rationalen Einsatz von Antibiotika gibt auch eine deutsche S3Leitlinie.

In Deutschland erfolgt die Mehrzahl der Antibiotikaverschreibungen im ambulanten Bereich (85\%). Hier war, wie aus einer Studie des Zentralinstituts für die Kassenärztliche Versorgung (ZI) hervorgeht, in den letzten Jahren speziell bei den über 70-Jährigen erfreulicherweise ein Rückgang zu verzeichnen. (eo)

Daneman $\mathrm{N}$ et al. Variability in Antibiotic Use Across Nursing Homes and the Risk of Antibiotic-Related Adverse Outcomes for Individual Residents. JAMA Intern Med 2015; online 29. Juni; doi: 10.1001/jamainternmed.2015.2770 